\title{
Análise das queixas da rede municipal encaminhadas para a Educação Especial
}

\author{
Bárbara Trevizan Guerra \\ Unesp - Bauru - São Paulo \\ Jéssica Aline Rovaris \\ Unesp - Bauru - São Paulo \\ Marília Mariano \\ Unesp - Bauru - São Paulo \\ Priscila Meireles Guidugli \\ Unesp - Bauru - São Paulo \\ Sofia Rosanti \\ Unesp - Bauru - São Paulo \\ Alessandra Turini Bolsoni-Silva \\ Unesp - Bauru - São Paulo
}

\begin{abstract}
Resumo
O Atendimento Educacional Especializado (AEE), em parceria com as escolas públicas, objetiva complementar a aprendizagem de alunos com deficiência, no contraturno da escola comum. Esta pesquisa objetivou descrever e analisar o conteúdo dos encaminhamentos dos professores das escolas públicas para o AEE, categorizando-o de acordo com o sexo, idade, diagnóstico de deficiência, problemas de comportamento externalizante e internalizante, dificuldade de aprendizagem e outros. Para tal contatou-se uma instituição que oferece o AEE em um município do interior paulista e foram analisados 92 encaminhamentos de crianças do ensino infantil. Os resultados demostram que a maior parte dos atendimentos realizados pelo AEE destina-se a crianças que não possuem diagnóstico de deficiência. Além disso, $69 \%$ das queixas referem-se a problemas de comportamento externalizantes. A partir de tais dados discute-se sobre práticas educativas de professores e alternativas de atendimento para alunos com ou sem deficiência que apresentam dificuldades no processo de ensino-aprendizagem.
\end{abstract}

Palavras-chave: Comportamento; educação especial; trabalho docente.

\section{Analysis of complaints of municipal directed network submitted to special education}

\begin{abstract}
The Atendimento Educacional Especializado (AEE), in partnership with public schools, aims complementary disabled student learning in the comum school, in turn against. This study aimed to describe and analyze the contents of the referrals of teachers in public schools to the AEE, categorizing them according to sex, age, disability diagnosis, behavior problems externalizing and internalizing, disabilities learning, and others. Was contacted an institution that offers the AEE, in an interior city of São Paulo, to describe and analyze 92 referrals of children of kindergarten. The results show that most of the services performed by the AEE intended for children that do not have disabilities. In addition, $69 \%$ of the complaints relate to externalizing behavior problems. From these data we discuss about educational practices of teachers, and alternative care for patients, whether or not students with disabilities, who have difficulties in the learning process.
\end{abstract}

Keywords: Behavior; special education; teaching work.

\section{Análisis de las quejas de la red municipal encaminadas para la Educación Especial}

\section{Resumen}

La Atención Educacional Especializada (AEE), en conjunto con las escuelas públicas, tiene como objetivo complementar el aprendizaje de alumnos con deficiencia, en el contra-horario de la escuela común. Esta investigación tiene el objetivo describir y analizar el contenido de los encaminamientos de los profesores de las escuelas públicas para el AEE, categorizándolo de acuerdo con el sexo, edad, diagnóstico de deficiencia, problemas de comportamiento exterior e interior, dificultad de aprendizaje y otros. Para eso se contactó una institución que ofrece el AEE en un municipio del interior paulista y se analizaron 92 encaminamientos de niños de la enseñanza infantil. Los resultados demostraron que la mayor parte de los atendimientos realizados por el AEE se destina a niños que no poseen diagnóstico de deficiencia. Además de eso, el $69 \%$ de las quejas se refieren a problemas de comportamiento externalizados. A partir de esos datos se discute sobre prácticas educativas de profesores y alternativas de atención para alumnos con o sin deficiencia que presentan dificultades en el proceso de enseñanza-aprendizaje.

Palabras clave: comportamiento; educación especial; labor docente. 


\section{Introdução}

Ao longo das últimas décadas diversas ações foram realizadas a fim de garantir o acesso ao ensino regular para crianças com deficiência ou necessidades educacionais especiais, tais como a Lei de Diretrizes e Bases da Educação Nacional (Lei $n^{\circ}$ 9.394, de 20 de dezembro de 1996) que conceituou a Educação Especial como modalidade de educação escolar, oferecida preferencialmente na rede regular de ensino, para educandos portadores de necessidades especiais (Lei $n^{\circ}$ 9.394, 1996, Capítulo V, Art. 58); o Programa de Educação Inclusiva: Direito à Diversidade em 2006, que visava melhorar a formação de gestores e educadores; e em 2007 o Plano de Desenvolvimento da Educação - PDE que também objetivou a formação de educadores e o acesso de todos à escolarização. Com tais políticas, passou a ocorrer uma gradual substituição da oferta de ensino para as escolares públicas regulares em detrimento das instituições de educação especial, que até então monopolizavam o ensino de tal população. A matrícula das pessoas com deficiência passou então a ocorrer em salas comuns das escolas públicas, e com isso houve o surgimento do Atendimento Educacional Especializado (AEE). Tal processo é observado no Decreto 6.571/2008, cuja Resolução n. ${ }^{\circ} 4$, de 2009, instituiu as Diretrizes Operacionais para o Atendimento Educacional Especializado:

Art. $1^{\circ}:(\ldots)$ os sistemas de ensino devem matricular os alunos com deficiência, transtornos globais do desenvolvimento e altas habilidades/superdotação nas classes comuns do ensino regular e no Atendimento Educacional Especializado (AEE), ofertado em salas de recursos multifuncionais ou em centros de Atendimento Educacional Especializado da rede pública ou de instituições comunitárias, confessionais ou filantrópicas.

Art. $2^{\circ}$ : OAEE tem como função complementar ou suplementar a formação do aluno por meio da disponibilização de serviços, recursos de acessibilidade e estratégias que eliminem as barreiras para sua plena participação na sociedade e desenvolvimento de sua aprendizagem (Brasil, 2009).

O AEE, portanto, caracteriza-se como uma peça fundamental para o processo de inclusão, pois se trata de uma ferramenta que pode auxiliar o aluno na inserção em escolas comuns, no processo de ensino e aprendizagem e, por consequência, auxiliar o trabalho do professor. Esses alunos, matriculados nas escolas comuns, poderão também desenvolver outras habilidades sociais uma vez que aumentam suas interações. No entanto, para além da reestruturação da legislação, há de se enfrentar as significativas mudanças na estruturação e no funcionamento do cotidiano escolar e, principalmente, na prática pedagógica. Diante desse cenário de inclusão, é importante discutir os comportamentos que os professores devem emitir a fim de promover a aprendizagem de seus educandos, bem como quais habilidades ele pode adotar para lidar com as especificidades de cada aluno.
Ao conjunto de habilidades sociais que objetivam a promoção de desenvolvimento e aprendizagem, em situação formal ou informal, Del Prette e Del Prette (2001) denominaram como Habilidades Sociais Educativas (HSE). Para um professor ser considerado socialmente competente, não basta a emissão de determinados comportamentos, mas é preciso considerar o aspecto funcional, uma vez que a ação do professor deve produzir efeitos, que probabilisticamente, gere mudanças no repertório dos educandos. Repertórios desenvolvidos de HSE promovem ao docente a formulação de estratégias pedagógicas que facilitam a interação com seus alunos, o que pode potencializar a capacidade desse profissional identificar a expressão de emoções e modelos adequados de comportamentos sociais e também promover a oportunidade de valorizar comportamentos mais adaptativos dos alunos, como tomar iniciativa, cooperar com colegas, dar feedback, lutar pelos próprios direitos, expressar sentimentos, negar pedidos e ter empatia, entre outros.

Além disso, o trabalho com as habilidades sociais de professores na escola remete à qualidade do processo de ensino e aprendizagem e a promoção de desenvolvimento humano dos alunos (Fonseca, 2012). No entanto, Castro e Bolsoni-Silva (2008), ao pesquisarem as relações entre professores e crianças, identificaram déficits de habilidades sociais interpessoais de educadores com relação ao desenvolvimento interpessoal dos alunos. Para Marturano e Loureiro (2003), é relevante discutir as práticas educativas dos professores, pois as escolas e as interações que lá ocorrem podem estabelecer e/ou manter comportamentos indesejáveis e déficits nas habilidades sociais infantis. Os professores com dificuldades em lidar com problemas de comportamento podem reforçar modelos inadequados, e/ou não promover modelos alternativos e mais adaptativos aos alunos (Fonseca, 2012; Mariano, 2011).

Apesar da falta de consenso quanto às definições, denominações, diagnóstico e tratamento de problemas de comportamento (Bolsoni-Silva, \& Del Prette, 2003), neste trabalho será utilizada a definição de Bolsoni-Silva (2003) que pontua que estes são déficits e/ou excessos comportamentais que prejudicam a interação da criança com pares e adultos, dificultam o acesso a novas contingências de reforçamento, que por sua vez, facilitariam a aquisição de repertórios relevantes de aprendizagem. No mesmo sentido, Achenbach e Edelbrock (1979) e Achenbach (1966), definem problemas de comportamento como padrões comportamentais que prejudicam as interações sociais e podem ser organizados como internalizantes, tais como retraimento, queixas somáticas, ansiedade, depressão e externalizantes, como por exemplo a agressividade, impulsividade, desobediência. Os problemas de comportamento internalizantes podem ser considerados como privados e desajustados, mas não ocorrem diretamente no ambiente, diferentemente dos externalizantes. Dessa forma, problemas de comportamento internalizantes, por exemplo, podem privar o acesso a diferentes interações sociais, e os externalizantes podem prejudicar relações interpessoais favorecendo conflitos (Bolsoni-Silva, Marturano, \& Manfrinato, 2005). Além disso, alguns autores 
destacam que os pais se preocupam mais com os problemas de comportamento externalizantes, por serem visíveis, do que com os internalizantes (Bolsoni-Silva, \& Marturano, 2002; Del Prette, \& Del Prette, 2005; Fonseca, 2012).

Ainda, Bolsoni-Silva, Marturano, Pereira e Manfrinato (2006) identificaram que crianças com problemas de comportamento teriam mais dificuldades para lidar com os desafios interpessoais no ambiente escolar do que em sua casa, pois o primeiro ambiente caracteriza-se como mais complexo e exigente. Como consequência, os problemas de comportamento intensificam-se na escola, uma vez que este ambiente oferece mais oportunidades de interação.

O estudo de Mariano (2011) que objetivou descrever práticas educativas de professores em lidar com o repertório infantil de crianças com ou sem problemas de comportamento apontou que os professores são mais habilidosos com crianças que não apresentam problemas de comportamento do que com as que apresentam. E, com crianças que os professores julgam ter problemas de comportamento, eles apresentam um padrão mais punitivo, pois (1) professores parecem reforçar, às vezes sem intenção, comportamentos problema, mesmo utilizando-se de práticas educativas habilidosas, que por consequência não promovem a diminuição dos problemas de comportamento dos alunos; (2) professores utilizam práticas diferentes entre os alunos que apresentam ou não problemas de comportamentos; (3) quanto à frequência de interação dos professores em diferentes situações, identificou-se que eles interagiram menos com as crianças com problemas de comportamento; (4) professores apresentaram práticas educativas inconsistentes, ora punindo comportamento inadequado, ora não.

Dados da revisão de literatura de Bolsoni-Silva, Villas Boas, Romera e Silveira (2010), entre 1986 e 2006, sobre intervenções para reduzir ou prevenir problemas de comportamento, demonstrou que somente $4,2 \%$ dos estudos incluíam avaliação e intervenção com professores. Assim, buscou-se descrever, analisar e discutir as queixas dos professores das escolas infantis e creches comuns, identificando os comportamentos com que eles apresentam dificuldades de lidar. Desta forma, objetivou-se descrever o conteúdo das queixas dos professores que encaminharam alunos para o Atendimento Educacional Especializado, caracterizando-as em relação à idade das crianças, sexo, deficiência e problemas de comportamento externalizante e internalizante.

\section{Método}

\section{Percurso amostral e procedimentos da coleta de dados}

A pesquisa foi realizada em uma instituição que oferece o AEE em parceira com a prefeitura de um município do centro-oeste paulista. Quando professores da rede de ensino municipal infantil consideram que seus alunos precisam de atendimento pedagógico especializado, preenchem uma ficha descrevendo as queixas e realizam o encaminhamento. Após a autorização da instituição para realização da pesquisa, foram transcritas na íntegra as queixas redigidas pelos professores, bem como os dados demográficos das crianças previstos no roteiro de coleta de dados. A amostra foi composta por 92 fichas de encaminhamentos de crianças matriculadas em escolas comuns que eram atendidas no AEE da instituição, seja para avaliação pedagógica, intervenção grupal ou individual ou aguardando atendimento, entre os meses de abril e maio de 2013. Foram excluídos os encaminhamentos que estavam na lista de espera para iniciar avaliação ou que haviam sido desligadas do apoio. Seguindo os preceitos éticos, são resguardadas as identidades das crianças encaminhadas e das instituições envolvidas.

\section{Instrumento}

Elaborou-se um roteiro de coleta das informações: idade da criança, data de nascimento, sexo, queixa escolar redigida pelo professor e diagnóstico/hipótese diagnóstica de alguma deficiência ou doença crônica.

\section{Procedimentos de tratamento e análise dos dados}

Para a categorização do conteúdo das queixas quanto aos problemas de comportamento, utilizou-se a descrição dos comportamentos da criança, sob o ponto de vista de seu professor, baseando-se no critério do "Child Behavior Checklist" (CBCL - Inventário de Comportamentos da Infância e Adolescência, Achenbach, 1991) para pré-escolares e escolares. Os dados foram organizados de acordo com a categorização para problema de comportamento externalizante e/ ou internalizante, comparando-os com a presença ou não de diagnóstico. Em tal categorização, cada descrição da queixa poderia conter mais de um problema de comportamento.

Tratando-se da análise das queixas foi utilizado o método qualitativo, abordando os dados além de sua expressividade numérica e o delineamento exploratório, enfatizando o caráter investigativo e descritivo (Baptista, \& Campos, 2007). As queixas foram definidas em categorias de acordo com a descrição do comportamento da criança, estabelecendo correlações entre as variáveis: idade, sexo, diagnóstico ou hipótese diagnóstica, problema de comportamento externalizante e/ou internalizante.

\section{Resultados}

Os resultados serão descritos considerando-se as variáveis: idade, sexo, ausência de diagnóstico, diagnóstico, suspeita de diagnóstico e queixa redigida pelo (a) professor (a), os quais são apresentados sequencialmente.

Em relação aos dados demográficos, observou-se que dentre as 92 fichas de encaminhamentos, 77 eram do sexo 
masculino, 15 do sexo feminino, com idade variando entre dois e seis anos. Assim, o maior número de encaminhamentos refere-se a crianças do sexo masculino (84\%), com quatro $(42,4 \%)$ e cinco (36\%) anos de idade, respectivamente.

Quanto ao diagnóstico ou hipótese diagnóstica, constatou-se que $81 \%$ das crianças encaminhadas ao AEE não possuíam diagnóstico, enquanto que $16 \%$ apresentaram diagnóstico fechado, e 3\% suspeita diagnóstica. Dentre os diagnósticos apresentados, observaram-se sete diagnósticos de Síndrome de Down, quatro de Transtorno do Espectro Autista e casos únicos de Hipotireodismo, Paralisia Cerebral, Hidrocefalia e Síndrome de Dand Walker e Hidrocefalia. Logo, o maior número de encaminhamentos foi realizado para crianças sem diagnóstico de deficiência, seguido daquelas com Síndrome de Down e do Transtorno do Espectro Autista. Nas suspeitas diagnósticas, houve duas para o Transtorno do Espectro Autista e uma indefinida, pois o professor suspeitou que a criança apresentava "alguma síndrome".

Tratando-se dos problemas de comportamento externalizante e internalizantes, serão apresentados para as crianças com diagnóstico e suspeita e sem diagnóstico, respectivamente nas Tabelas 1 e 2 .

Tabela 1. Descrição dos problemas de comportamento externalizante apresentados para crianças com e sem diagnóstico.

\begin{tabular}{|c|c|c|}
\hline $\begin{array}{l}\text { Comportamento } \\
\text { externalizante }\end{array}$ & $\begin{array}{l}\text { Criança sem } \\
\text { diagnóstico }\end{array}$ & $\begin{array}{c}\text { Criança } \\
\text { com } \\
\text { diagnóstico }\end{array}$ \\
\hline $\begin{array}{l}\text { Desatenção/dificuldade de } \\
\text { concentração }\end{array}$ & 16 & 3 \\
\hline Problema em seguir regra & 14 & 2 \\
\hline Agressividade & 13 & 2 \\
\hline Agitação & 9 & 2 \\
\hline Inquieto & 3 & 0 \\
\hline Bater & 2 & 0 \\
\hline Jogar-se ou rolar no chão & 1 & 1 \\
\hline Grita & 1 & 3 \\
\hline Quer atenção só para ele & 1 & 0 \\
\hline Anda pela sala & 1 & 0 \\
\hline Toma brinquedos das crianças & 1 & 0 \\
\hline Impulsivo & 1 & 0 \\
\hline Fala alto & 1 & 0 \\
\hline Nervosismo & 1 & 0 \\
\hline Birra & 1 & 1 \\
\hline $\begin{array}{c}\text { Auto-lesivo (arrancar cabelo, } \\
\text { bater a cabeça no chão) }\end{array}$ & 0 & 2 \\
\hline Fixação por objeto & 0 & 1 \\
\hline $\begin{array}{l}\text { Total de comportamentos } \\
\text { externalizantes }\end{array}$ & 66 & 17 \\
\hline
\end{tabular}

Tabela 2. Descrição dos problemas de comportamento internalizantes apresentados para crianças com e sem diagnóstico.

\begin{tabular}{ccc}
\hline $\begin{array}{c}\text { Comportamento } \\
\text { internalizante }\end{array}$ & $\begin{array}{c}\text { Criança sem } \\
\text { diagnóstico }\end{array}$ & $\begin{array}{c}\text { Criança com } \\
\text { diagnóstico }\end{array}$ \\
\hline
\end{tabular}

22

social; fala pouco

\begin{tabular}{lll}
\hline Chora & 7 & 1 \\
\hline
\end{tabular}

Não expressa

os sentimentos dificuldade de expressar sentimentos; instropecção

\section{Total de comportamentos} internalizantes

7

De acordo com as Tabelas 1 e 2, a análise dos encaminhamentos de crianças sem diagnóstico identifica: a) queixas exclusivamente internalizantes: 11 para o sexo masculino e 2 para o sexo feminino; b) queixas exclusivamente externalizantes: 24 para o sexo masculino e 4 para o sexo feminino; e c) queixas internalizantes e externalizantes: 9 para o sexo masculino e 1 para o sexo feminino. Ainda para esse grupo, as professoras indicaram outros problemas, tais como: a) biológicos (15) - déficit no controle esfincteriano, salivação excessiva/controle da saliva, problemas com acuidade visual, sonolência e histórico de convulsões; b) problemas no desenvolvimento da linguagem (43); c) solicitação da professora para avaliação e intervenção na instituição (12); d) problemas familiares/suporte social (4); e) baixo desempenho acadêmico/deficiência acadêmica (4); f) solicitação da família para atendimento (3); e g) outros (10) - queixas que não descreviam o comportamento (ex: "Apresenta dificuldades nas atividades relacionadas à sua faixa etária"). No grupo de crianças com deficiência, foram apresentadas outras queixas: a) biológicas (11) - atraso no desenvolvimento motor, salivação excessiva/controle da saliva, problema com acuidade visual, déficit no controle esfincteriano, lentidão, histórico de convulsões, refluxo na bexiga, má formação/deficiência física, sonolência; b) problemas no desenvolvimento da linguagem (8); c) solicitação da professora para avaliação e intervenção na instituição (4); d) baixo desempenho acadêmico/deficiência acadêmica (1); e) outros (11) - queixas que não descreviam o comportamento. O encaminhamento de tais queixas demonstra certa dificuldade dos professores para estabelecerem critérios para encaminhar as crianças para o AEE, pois parecem supor que tal apoio lidará com questões além das pedagógicas, como 
desenvolvimento motor, salivação excessiva, problema no desenvolvimento da linguagem, além de queixas que não descrevem o comportamento problema.

Quanto à queixa predominante em crianças com diagnóstico, observou-se que: a) queixas exclusivamente internalizantes: uma para o sexo masculino; b) queixas exclusivamente externalizantes: uma para o sexo masculino e uma para o sexo feminino; c) queixas internalizantes e externalizantes: três para ao sexo masculino e uma para o sexo feminino. O percentil de queixas de comportamentos externalizantes que aparecem nas categorias das crianças já diagnosticadas é cerca de $41 \%$ maior do que para os internalizantes para a mesma população, resultado semelhante ao encontrado para as crianças sem diagnóstico. Assim, a maior parte dos problemas externalizantes se repete para as duas populações, e houve predomínio de queixas exclusivamente externalizantes e combinadas (internalizante e externalizante) para o sexo masculino. Não houve diferença para o grupo de crianças atípicas em relação às queixas somente de comportamento externalizante.

\section{Discussão}

Os dados descritos em relação às crianças encaminhadas e atendidas no AEE sem deficiência conflitam com as diretrizes legais, uma vez que o AEE é especificamente direcionado para crianças com deficiência, transtornos globais do desenvolvimento e/ou altas habilidades/superdotação (Brasil, 2009). Diante da incongruência entre a legislação e a prática observada na parceria entre as instituições de ensino regular e ensino especializado, levantam-se como hipóteses: (1) as crianças com deficiência, transtornos globais do desenvolvimento e/ou altas habilidades/superdotação não estão sendo matriculadas nas escolas de ensino comum e, portanto, não são encaminhadas ao AEE para a complementação; (2) tais crianças estão sendo matriculadas e os respectivos professores não estão fazendo os encaminhamentos para o AEE no contraturno, pois eles possuem habilidades educativas suficientes. No entanto, esta última hipótese parece conflitante com as demais queixas, uma vez que estas descrevem a necessidade de suplementação de aprendizagem de crianças que não possuem diagnóstico de deficiência, mas aparentemente apresentam problemas de comportamento, conforme especificado nas Tabelas 1 e 2.

Quanto aos problemas de comportamento, o alto número de queixas de comportamento externalizante pode ser devido à dificuldade que crianças encontram no contexto escolar, por ser um ambiente que exige mais habilidades (Bolsoni-Silva, \& cols., 2006). Além disso, problemas de comportamento externalizantes são observados de forma direta, causando maior incômodo social, diferentemente dos internalizantes (Achenbach, 1966; Achenbach, \& Rescorla, 2000; Bandeira, Rocha, Souza, Del Prette, \& Del Prette, 2006; Dornelles, Susin, \& Oliveira, 2009) que se manifestam na relação do indivíduo com ele próprio, e muitas vezes não são identificados pelas pessoas que convivem com a crian- ça, em especial por que são considerados mais sociáveis (Achenbach, 1966; Bolsoni-Silva, \& Del Prette, 2003).

Tal dado é relevante, uma vez que se observa que problemas de comportamento internalizantes produzem consequências prejudiciais no desenvolvimento infantil que perduram até a idade adulta, como a baixa autoestima, dificuldades acadêmicas, prejuízos/déficits nos relacionamentos interpessoais, problemas de saúde mental crônicos - fobia social, depressão, abuso de substâncias e pensamentos suicidas (Merrell, \& Harlacher, 2008). Nos problemas de comportamento internalizantes, cerca de $85,7 \%$ das queixas apresentadas referem-se à dificuldade de socialização: isolamento social, falar pouco, não interagir. Particularmente em relação à comunicação, Bolsoni-Silva e Marturano (2006) indicam que a capacidade de conversação é relevante para o desenvolvimento das interações sociais, como um repertório que promove o desenvolvimento de outras habilidades e previne o surgimento de problema de comportamento, posto que possibilitam às crianças interações positivas com os pares, pais e professores (Bolsoni-Silva, 2003).

Os problemas identificados como biológicos aparecem em menor quantidade para ambas as populações, se comparados às demais categorias de análise. Disso, supõe-se que parte das crianças não diagnosticadas pela instituição pode apresentar problemas apenas de ordem comportamental, tais como dificuldade em seguir regras, comportamentos de birra, andar pela sala, pegar o brinquedo dos colegas, entre outros. Também foram indicados alguns comportamentos categorizados em Outros, como "quando chora a face entorta", "usa mamadeira", "anda na ponta dos pés", "necessita de supervisão".

Esse tipo de descrição não deixa claro qual o problema de comportamento da criança; no entanto, pode sinalizar a dificuldade dos professores em identificar quais comportamentos indicam que a criança precisa de um atendimento especializado, quais são resolvidos por meios de práticas educativas direcionadas ao problema de comportamento apresentado e quais aspectos são esperados de acordo com a fase de desenvolvimento infantil. Ao encaminhar de forma pouco criteriosa os alunos, os professores parecem descrever queixas incompatíveis com o objetivo do AEE, que visa a complementação da formação acadêmica do aluno, auxiliando o professor na promoção de aprendizagem e desenvolvimento deste (Brasil, 2009).

Alguns autores (Ferreira, \& Marturano, 2002) já discutiam que uma visão organicista dos problemas de comportamento poderia estimular o encaminhamento da criança ao ensino especial, inclusive por atribuir suas causas à própria criança. Diante disso, é importante considerar a causalidade múltipla de tais comportamentos e a respectiva função no ambiente que este possui, uma vez que o contexto em que a criança está inserida não supre suas demandas (Sidman, 1995; Bolsoni-Silva, \& Del Prette, 2003; Meyer, Del Prette, Zamignani, Banaco, \& Tourinho, 2010).

Em relação às queixas apresentadas, destaca-se que cerca de $20 \%$ referem-se às especificidades da deficiência da criança, associadas ou não a problemas de comporta- 
mento. Apesar de muitas deficiências tornarem-se um possível complicador para o processo de ensino-aprendizagem, o que justificaria o encaminhamento aos serviços especializados como o AEE, ressalta-se que nem todas as crianças diagnosticadas como deficientes precisam deste atendimento. No entanto, certos professores promovem a educação de forma homogeneizada, desconsiderando as especificidades de cada criança, em especial do aluno com desenvolvimento atípico (Fabrício, Souza, \& Gomes, 2007). Logo, tende-se a aumentar o número de encaminhamentos de alunos com necessidades educacionais especiais, utilizando a própria deficiência como justificativa e esquecendo-se de ponderar que o ensino comum poderia se adequar para atender às respectivas demandas.

Portanto, torna-se necessário identificar no contexto escolar quais comportamentos dos professores podem funcionar para não estabelecer, intensificar e/ou manter padrões comportamentais desadaptados. Discute-se que professores devem promover e contribuir para o desenvolvimento das habilidades sociais das crianças, a fim de que tal repertório possa atuar como protetor ao risco de transtornos psíquicos, reduzindo-o e/ou eliminando-o. Além disso, o desenvolvimento de habilidades sociais permite a aprendizagem de maneira mais adaptativas de interações, ao invés de somente eliminar formas desadaptadas (Hudson, Flannery-Schroeder, \& Kendall, 2004). Assim, a escola possui um papel fundamental no desenvolvimento das crianças, podendo atuar na prevenção de problemas de comportamento e outros transtornos (Marturano, \& Loureiro, 2003; Gomide, 2003).

Outro aspecto que deve ser considerado refere-se à consistência nas práticas educativas de professores, pois Bolsoni-Silva e cols. (2006) levantaram como hipótese que como a criança muda seu comportamento em função do ambiente em que está, os educadores podem consequenciar e discriminar diferentemente comportamentos socialmente habilidosos, promovendo uma inconsistência na prática educativa. Inconsistências em tais práticas educativas tendem a estabelecer e/ou manter padrões de birra e desobediência.

Por fim, considerando-se a relevância social das parcerias entre os AEE e as escolas regulares que recebem alunos portadores de deficiência, de transtornos do desenvolvimento global e com altas habilidades, discute-se a necessária valorização e formação do professor como um importante agente de promoção de aprendizagens e desenvolvimento humano. Assim, em uma política de educação inclusiva, ressalta-se o necessário esclarecimento deste profissional sobre a função do AEE, a fim de que ele encaminhe casos específicos, compatíveis com os serviços que o programa está apto a realizar.

Como as investigações desta pesquisa apresentam um caráter documental, baseadas na descrição de queixas de professores, não foi possível obter maiores esclarecimentos sobre algumas descrições de comportamentos vagas e confusas. Novas pesquisas poderão ser desenvolvidas para investigar os motivos do número reduzido de crianças com diagnóstico de deficiência matriculadas no AEE, com- parando tal dado com o número de crianças matriculadas no sistema educacional do município. Assim, será possível identificar se há crianças com deficiência sem encaminhamento e as respectivas razões. Sugere-se a futuras pesquisas o cruzamento de diferentes fontes de dados, como por exemplo, delinear um método que utilize além do discurso do professor, observações nas instituições envolvidas.

\section{Referências}

Achenbach, T. M. (1966). The classification of children's psychiatric symptoms: a factor-analytic study. Psychological monographs general and applied, 80(7), 1-37.

Achenbach, T. M. (1991). Manual for the Child Behavior Checklist/ 4-18 and 1991 profile. EUA: University of Vermont.

Achenbach, T. M., \& Edelbrock, C. S. (1979). The child behavior profile: II. Boys aged 12-16 and girls aged 6-11 and 12-16. Journal of consulting and clinical psychology, 47(2), 223-233.

Achenbach, T. M., \& Rescorla, L. A. (2000). Manual for ASEBA preschool forms \& profiles. Burlington, University of Vermont, Research Center for Children, Youth, \& Families.

Bandeira, M., Rocha, S. S., Souza, T. M. P., Del Prette, Z. A. P., \& Del Prette, A. (2006). Comportamentos problemáticos em estudantes do ensino fundamental: características da ocorrência e relação com habilidades sociais e dificuldades de aprendizagem. Estudos de Psicologia, 11(2), 199-208.

Baptista, M. N., \& Campos, D. C. (2007). Metodologias de pesquisa em ciências: análises quantitativa e qualitativa. Rio de Janeiro: LTC.

Brasil (2009). Resolução CNE/CEB 04 de 02 de outubro de 2009. Diretrizes operacionais para o atendimento educacional especializado na educação básica, modalidade educação especial. Brasília, DF: Ministério da Educação.

Bolsoni-Silva, A. T. (2003). Habilidades Sociais Educativas, Variáveis Contextuais e Problemas de Comportamento: comparando pais e mais de pré-escolares. Tese de Doutorado, Universidade de São Paulo, Ribeirão Preto-SP.

Bolsoni-Silva, A. T., \& Del Prette, A. (2003). Problemas de Comportamento: um panorama da área. Revista Brasileira de Terapia Comportamental e Cognitiva, 5(2), 91-103.

Bolsoni-Silva, A. T., \& Marturano, E. M. (2002). Práticas Educativas e Problemas de Comportamento: uma análise a luz das habilidades sociais. Estudos de Psicologia, 7, 227-235.

Bolsoni-Silva, A. T., \& Marturano, E. M. (2006). Aqualidade da interação pais e filhos e sua relação com problemas de comportamentos de pré-escolares. Em M. Bandeira, Z. A. P. Del Prette, \& A. Del Prette 
(Orgs.), Estudos sobre Habilidades Sociais e Relacionamento Interpessoal. São Paulo: Casa do Psicólogo.

Bolsoni-Silva, A. T., Marturano, E. M., \& Manfrinato, J. W. S. (2005). Mães avaliam comportamentos socialmente "desejados" e "indesejados" de pré-escolares. Psicologia em Estudo, 10(2), 245252.

Bolsoni-Silva, A. T., Marturano, E. M., Pereira, V. A, \& Manfrinato, J. W. S. (2006). Habilidades Sociais e Problemas de Comportamento de Pré-Escolares: Comparando Avaliações de Mães e de Professoras. Psicologia: Reflexão e Crítica, 19(3), 460-469.

Bolsoni-Silva, A. T., Villas Boas, A. C. V., Romera, V. B., \& Silveira, F. F. (2010). Caracterização de programas de intervenção com crianças e/ou adolescentes. Arquivos Brasileiros de Psicologia, $62,104-114$

Castro, A. B., \& Bolsoni-Silva, A. T. (2008). Habilidades Sociais na educação: relações entre concepções e práticas docentes na educação infantil. Em V. L. F. Capellini, \& R. M. Manzoni, R. M. (Orgs.), Políticas públicas, práticas pedagógicas e ensinoaprendizagem: diferentes olhares sobre o processo educacional. São Paulo: Cultura Acadêmica.

Del Prette, Z. A. P., \& Del Prette, A. (2001). Psicologia das relações interpessoais: vivências para o trabalho em grupo. Petrópolis: Vozes.

Del Prette, Z. A. P., \& Del Prette, A. (2005). Psicologia das habilidades sociais na infância: teoria e prática. Petrópolis: Vozes.

Del Prette, Z. A. P., \& Del Prette, A. (2006). Treinamento em Habilidades Sociais; o método vivencial e a participação do professor. Em M. Bandeira, Z. A. P. Del Prette, \& A. Del Prette (Orgs.), Estudos sobre habilidades sociais e relações interpessoais. São Paulo: Casa do Psicólogo.

Dornelles, C., Susin, N., \& Oliveira, M. S. (2009). Comportamentos internalizantes e externalizantes em adolescentes atendidos em clínica-escola. Trabalho apresentado no X Salão de Iniciação Científica da PUC, Porto Alegre - Rio Grande do Sul.

Fabrício, N. M. C., Souza, V. C. B., \& Gomes, E. E. A. S. (2007). Perfil do Professor Inclusivo. Revista Psicopedagogia, 24(74), 117-25.

Ferreira, M. C. T., \& Marturano, E. M. (2002). Ambiente familiar e os problemas de comportamento apresentados por crianças com baixo desempenho escolar. Psicologia: Reflexão e Crítica, 15(1), 35-44.

Fonseca, B. C. R. (2012). Práticas Educativas de genitores e professoras e repertório comportamental de crianças do ensino fundamental: estudos de caso. Dissertação de Mestrado, Universidade Estadual Paulista, Bauru - SP.

Gomide, P. I. C. (2003). Estilos parentais e comportamento antisocial. Em A. Del Prette, \& Z. A. P. Del Prette (Orgs.), Habilidades sociais, desenvolvimento e aprendizagem: Questões conceituais, avaliação e intervenção. Campinas: Alínea.

Hudson, J. L., Flannery-Schroeder, E., \& Kendall, P. C. (2004). Primary Prevention of Anxiety Disorders. Em D. J. A. Dozois, \& K. S. Dobson (Orgs.), The prevention of anxiety and depression. Theory, research and practice. Washington: American Psychological Association.

Lei n. 9.394, de 20 de dezembro de 1996. (1996, 23 de dezembro). Estabelece as Diretrizes e Bases da Educação Nacional. Diário Oficial da União, seção 1.

Mariano, M. L. (2011). Análise de práticas educativas de professores na interação com alunos com e sem problemas de comportamentos. Dissertação de Mestrado, Universidade Estadual Paulista, Bauru $-\mathrm{SP}$.

Marturano, E. M., \& Loureiro, S. O. (2003). O desenvolvimento emocional e as queixas escolares. Em Z. A. P. Del Prette, \& A. Del Prette (Orgs.), Habilidades sociais, desenvolvimento e aprendizagem: questões conceituais, avaliação e intervenção. Campinas: Alínea.

Merrell, K. W., \& Harlacher, J. E. (2008). Behavior rating scales. Em R. P. Archer, \& S. R. Smith (Orgs.), Personality assessment. New York: Routledge/Taylor \& Francis.

Meyer, S. B., Del Prette, G., Zamignani, D. R., Banaco, R. A., \& Tourinho, E. Z. (2010). Análise do Comportamento e Terapia Analítico-Comportamental. Em E. Z. Tourinho, \& S. V. Luna (Orgs.), Análise do comportamento: investigações históricas, conceituais e aplicadas, São Paulo: Rocca.

Sidman, M. (1995). Coerção e suas implicações. Campinas: Psy.
Enviado em: 09/01/2015

Reformulado em: 13/01/2015

Aprovado em: 12/03/2015 


\section{Sobre as autoras}

Bárbara Trevizan Guerra (barbaratrevizan@hotmail.com)

Psicóloga e Mestranda no Programa de Pós-Graduação em Psicologia do Desenvolvimento e Aprendizagem / Unesp Bauru.

Jéssica Aline Rovaris (jessica.rovaris@gmail.com)

Psicóloga e Mestranda no Programa de Pós-Graduação em Psicologia do Desenvolvimento e Aprendizagem / Unesp Bauru.

Marília Mariano (mariano.mrl@gmail.com)

Psicóloga e Mestranda no Programa de Pós-Graduação em Psicologia do Desenvolvimento e Aprendizagem / Unesp Bauru.

Priscila Meireles Guidugli (pity_mg@yahoo.com.br)

Psicóloga e Mestranda no Programa de Pós-Graduação em Psicologia do Desenvolvimento e Aprendizagem / Unesp Bauru.

Sofia Rosanti (sofyrosanti@yahoo.com.br)

Pedagoga e Mestranda no Programa de Pós-Graduação em Psicologia do Desenvolvimento e Aprendizagem / Unesp Bauru.

Alessandra Turini Bolsoni-Silva (bolsoni@fc.unesp.br)

Professora Livre Docente na Faculdade de Ciências e Programa de Pós-Graduação em Psicologia do Desenvolvimento e Aprendizagem / Unesp Bauru.

O trabalho apresentado neste manuscrito foi realizado na Associação de Pais e Amigos do Excepcional na cidade de Bauru - SP. Tal instituição possui um programa de Apoio Educacional Especializado que atende crianças encaminhadas pela Rede de Ensino Municipal e foi colaboradora para a análise dos dados. Previamente ao acesso aos dados, a diretora responsável autorizou via documento a realização da pesquisa. 